\title{
Le traitement antiviral suppressif du virus herpès simplex prévient-il la transmission du virus chez une population séropositive pour le VIH? Examen systématique
}

\author{
Smith $\mathrm{CR}^{1,2^{*}}$, Pogany $\mathrm{L}^{1}$, Auguste $\mathrm{U}^{1}$, Steben $\mathrm{M}^{3}$, Lau TTY ${ }^{4}$
}

\section{Résumé}

Contexte : Il a été démontré que, chez les personnes atteintes d'une infection génitale au virus herpès simplex (VHS), la co-infection au virus de l'immunodéficience humaine (VIH) augmente la fréquence et la gravité des symptômes du VHS, I'élimination du VHS et le risque de transmission du VHS.

Objectif : Déterminer si le traitement antiviral suppressif de l'infection génitale au VHS chez une population séropositive pour le VIH prévient la transmission du VHS aux partenaires réceptifs.

Méthodologie : Une recherche systématique de la littérature a été effectuée dans les bases de données MEDLINE et EMBASE pour répertorier les essais comparatifs randomisés dont les résultats ont été publiés entre janvier 2005 et juin 2015. Les critères d'inclusion étaient les articles en anglais ou en français décrivant des traitements antiviraux suppressifs du VHS. Les études devaient rapporter les résultats sur la transmission du VHS dans les populations séropositives pour le VIH. Les marqueurs de substitution du risque de transmission du VHS, telles la détection et la charge virale du VHS, ont aussi été inclus. Les articles ont fait l'objet d'une évaluation du risque de biais, et ceux dont le risque de biais était faible ont fait l'objet d'une extraction de données afin de réaliser une synthèse descriptive.

Résultats : Cet examen a permis de répertorier 13 articles parmi lesquels 1 seule étude a mesuré directement la transmission du VHS. Le taux de transmission global était $<10 \%$ et le traitement antiviral suppressif n'a entraîné aucun effet de protection significatif (taux de transmission de $9 \%$ dans le groupe acyclovir par rapport à $6 \%$ dans le groupe placebo; taux de risque : 1,35, IC de $95 \%: 0,83$ à 2,20). Les 12 autres articles traitaient des marqueurs de substitution du risque de transmission, soit la détection et la charge virale du VHS. Le traitement suppressif par l'acyclovir semble efficace pour réduire la détection du VHS chez les populations séropositives pour le VIH, mais il ne semble pas réduire la charge virale. Le traitement suppressif par le valacyclovir peut être efficace pour réduire la détection et la charge virale du VHS chez les patients séropositifs pour le VIH qui n'ont jamais reçu un traitement antirétroviral, mais son effet semble s'annuler chez ceux qui reçoivent un traitement antirétroviral simultanément.

Conclusion : D'après les données actuelles, le traitement antiviral suppressif peut réduire la détection et la charge virale du VHS, mais son effet sur la transmission du VHS demeure obscur. Les cliniciens doivent mettre en garde les patients séropositifs pour le VIH infectés par le VHS que le traitement suppressif pourrait ne pas réduire le risque de transmission du VHS aux partenaires réceptifs.

\section{Affiliations}

${ }^{1}$ Centre de la lutte contre les maladies transmissibles et les infections, Direction générale de la prévention et du contrôle des maladies infectieuses, Agence de la santé publique du Canada, Ottawa (Ontario)

2Dalla Lana School of Public Health, Université de Toronto, Toronto (Ontario)

3Unité des infections transmissibles sexuellement, Institut national de santé publique du Québec, Montréal (Québec)

${ }^{4}$ Pharmaceutical Sciences, Vancouver General Hospital, Vancouver Coastal Health et Faculté des Sciences pharmaceutiques, Université de la Colombie-Britannique, Vancouver (Colombie-Britannique)

${ }^{\star}$ Correspondance : courtneyrady. smith@mail.utoronto.ca
Citation proposée : Smith CR, Pogany L, Auguste U, Steben M, Lau TTY. Le traitement antirviral suppressif du virus herpès simplex prévient-il la transmission du virus chez une population séropositive pour le VIH? Examen systématique. Relevé des maladies transmissibles au Canada. 2016;42-2:41-50.

https://doi.org/10.14745/ccdr.v42i02a03f 


\section{Introduction}

Environ $14 \%$ des Canadiens adultes ont obtenu un résultat positif au test de dépistage de linfection génitale au VHS de type 2 (VHS-2) en 2009 (1). Le VHS est particulierement repandu chez les por lon 2009 (1). Le VHS est $p$

Il a été démontré que la réactivation de l'infection génitale au VHS accroit la charge virale du VIH, augmentant ainsi le risque de transmission et de progression de l'infection au VIH $(3,4)$. Il a été démontre que le VIH, à son tour, augmente la du VHS et le risque de transmission du VHS $(5,6)$. Compte du VHS et le risque de transmission du VHS $(5,6)$. Compte
tenu de cette interaction entre le VHS et le VIH, la prévention de la transmission du VHS chez les populations séropositives pour le VIH représente une préoccupation de santé publique importante. La réduction au minimum des taux de co-infection au VHS chez les personnes séropositives pourrait prévenir Vaugmentation de la charge virale du $\mathrm{VH}$, la transmission du au VIH et au VHS. La prévention de la transmission du VHS des partenaires séropositifs pour le $\mathrm{VIH}$ aux partenaires séronégat est également importante sur le plan de la santé publique, puisque I'on estime que linfection au VHS triple le risque contracter le $\mathrm{VIH}$ (7).

Le traitement suppressif par l'acyclovir, le famciclovir ou le valacyclovir est couramment utilisé chez les populations co-infectées par le VIH et le VHS. Ces agents se sont révélés ce qui a été associé à un risque réduit de transmission du VHS chez les populations immunocompetentes $(8,9)$. Une question digne d'interêtét est celle de savoir si ces agents réduisent aussi le pour le VIH. Les plus récents examens ont été publiés en 2007 $(10,11)$, mais aucun d'eux n'a permis de répertorier suffisamm de publications pour évaluer la transmission du VHS chez les populations séropositives pour le VIH.

L'objectif de cet examen systématique visait à évaluer les essais comparatifs randomisés menés auprès de populations co-infectées par le VIH et le VHS en mettant l'accent sur l'effet des traitements antiviraux suppressifs du
la détection et la charge virale du VHS.

\section{Méthodologie}

\section{Stratégie de recherche}

Une recherche systématique dans les bases de données MEDLINE et EMBASE a eté effectuée pour répertorier des articles sur les essais comparatifs randomisés dont les résultats ont été publiés au cours des 10 demieres annés. Les examen précédents comprenaient les recherches de documents publiés termes MeSH pour " résultats de traitement " et "virus herpes simplex » ainsi que des mots-clés pertinents (voir l'encadré).

\begin{tabular}{|l|l|}
\hline No & Stratégie de recherche \\
\hline 1 & résultats de traitement exp./ \\
\hline 2 & efficacité du médicament/ ou effet du médicament/ \\
\hline 3 & (effic.* ou résultat ${ }^{\star}$ ).mp. \\
\hline 4 & 1 ou 2 ou 3 \\
\hline 5 & $\begin{array}{l}\text { essai exp. *herpès simplex / ou (herpès ou VHS1 ou VHS2 ou } \\
\text { VHS-1 ou VHS-2 ou VHS).tw. }\end{array}$ \\
\hline 6 & 4 et 5 \\
\hline 7 & limite 6 à (essai contrôlé randomisé et 10 dernières années) \\
\hline
\end{tabular}

\section{Critères d'admissibilité}

Les articles étaient admissibles s'ls étaient rédiges en français ou en anglais et s'ils avaient été publiés entre janvier 2005 et juin 2015 dans des revues examinees par des pairs. Les études devaient porter sur r infection genitale au VHS chez les dultes (18 ans et plus) séropositifís pour le VH et decrire un essai contrốé randomisé́. Les articles ont été inclus seulement si, aux fins de comparaison pharmacologique, l'évaluation avait porté sur un traitement suppressif par au moins l'un des trois agents oraux anti-VHS les plus fréquemment utilisés, soit 'acyclovir, le famciclovir ou le valacyclovir. Tous les articles admissibles devaient faire état de résultats tels que la ayant évalú la destection ou la charge virale du VHS. Les etudes ont été exclues du fait qu'elles n'étaient accessibles que dans contexte de recherches cliniques et que les traitements avaient une efficacité limitée. Les études chez les femmes enceintes ainsi que les études ayant porté sur les traitements episodiques

es titres ont été évalués et exclus s'ils n'avaient aucun lien avec le VHS et le VIH. Les articles satisfaisant à ces critères de sélection de base ont eté obtenus aux fins d'examen du texte integral et evalués de façon indépendante par deux auteurs (CRS é UA) d'apres les criteres d'nclusion décrits

exper examinées et récupérées, le cas échéant. Une évaluation formelle de la qualité de chaque article répondant à tous les criteres d inclusion a été effectuee de façon indépendante par deux auteurs (CRS et UA) au moyen de l'outil de la Cochrane Collaboration pour évaler moyen de loutl de la Coc

\section{Extraction des données}

Les données ont été résumées par un auteur (CRS) dans un d'exh d'exhaustivité par un autre auteur (UA). Les donnees pertinentes pays où l'étude a été menée, les traitements pharmacologiques, la période de suivi, l'administration ou non d'un traitement
Tableau 1 : Caractéristiques d'un traitement antiviral suppressif du virus herpès simplex chez une population seropositive pour le VIH

\begin{tabular}{|c|c|c|c|c|c|c|}
\hline Auteurs & Nombre & $\begin{array}{c}\text { Pays où } \\
\text { l'étude a été } \\
\text { menée }\end{array}$ & $\begin{array}{c}\text { Traitement } \\
\text { pharmacologique }\end{array}$ & $\begin{array}{l}\text { Mois de } \\
\text { suivi }\end{array}$ & Traitement du VIH & $\begin{array}{c}\text { Mesure des } \\
\text { résultats }\end{array}$ \\
\hline \begin{tabular}{|l} 
Mujugira et al. \\
(14) 2013 .
\end{tabular} & \begin{tabular}{|l}
911 hommes et \\
femmes
\end{tabular} & $\begin{array}{l}7 \text { (pays de l'est et } \\
\text { du sudd } \\
\text { de l'Arique) } \\
\end{array}$ & $\begin{array}{l}\text { Acyclovir (400 mg bid) par } \\
\text { rapport au placebo }\end{array}$ & 24 & \begin{tabular}{|l|}
$\begin{array}{l}\text { Ne recevaient pas de } \\
\text { traitement antirétroviral }\end{array}$ \\
\end{tabular} & Transmission du VHS-2 \\
\hline \begin{tabular}{|l|} 
Baeten et al. \\
(15) 2008
\end{tabular} & 20 femmes & Pérou & $\begin{array}{l}\text { Valacyclovir (500 mg bid) par } \\
\text { rapport au placebo }\end{array}$ & 2 & \begin{tabular}{|l|l} 
Ne recevaient pas de \\
traitement antirétroviral
\end{tabular} & $\begin{array}{l}\text { Détection de l'infection } \\
\text { génitale au VHS-2 } \\
\text { Charge virale de l'infection } \\
\text { génitale au VHS-2 } \\
\text { Événements indésirables }\end{array}$ \\
\hline $\begin{array}{l}\text { Cowan et al. } \\
\text { (16) } 2008 \text {. }\end{array}$ & 125 femmes & Zimbabwe & $\begin{array}{l}\text { Acyclovir (400 mg bid) par } \\
\text { rapport au placebo }\end{array}$ & 3 & $\begin{array}{l}\text { Non explicitement indiqué } \\
\text { (le tratitement antiriteroiral } \\
\text { était toutefois rarementr } \\
\text { accessible au moment de } \\
\text { l'étude) }\end{array}$ & $\begin{array}{l}\text { Détection de linfection } \\
\text { genitale au VHS-2 }\end{array}$ \\
\hline $\begin{array}{l}\text { Delany et al. } \\
\text { (17) } 2009 \text {. }\end{array}$ & 300 femmes & Afrique du Sud & $\begin{array}{l}\text { Acyclovir (400 mg bid) par } \\
\text { rapport au placebo }\end{array}$ & 3 & \begin{tabular}{|l} 
Ne recevaient pas de \\
traitement antirétroviral
\end{tabular} & $\begin{array}{l}\text { Détection de l'infection } \\
\text { génitale au VHS-2 } \\
\text { Charge virale de l'infection } \\
\text { génitale au VHS-2 } \\
\text { Evénements indésirables }\end{array}$ \\
\hline $\begin{array}{l}\text { Dunne et al. } \\
\text { (18) } 2008 \text {. }\end{array}$ & 67 femmes & Thaillande & $\begin{array}{l}\text { Acyclovir (800 mg bid) par } \\
\text { rapport au placebo }\end{array}$ & 1 & $\begin{array}{l}\text { Ne recevaient pas de } \\
\text { traitement antirétroviral }\end{array}$ & $\begin{array}{l}\text { Détection de l'infection } \\
\text { génitale au VHS-2 }\end{array}$ \\
\hline \begin{tabular}{|l|} 
Kim et al. (19) \\
2010
\end{tabular} & $\begin{array}{l}76 \text { hommes et } \\
\text { femmes }\end{array}$ & \begin{tabular}{|l} 
Afrique du Sud, \\
Zimbabwe, \\
Zambie, Pérou, \\
Etats-Unis \\
\end{tabular} & $\begin{array}{l}\text { Acyclovir (400 mg bid) par } \\
\text { rapport au placebo }\end{array}$ & 6 & \begin{tabular}{|l|}
$\begin{array}{l}\text { Ne recevaient pas de } \\
\text { traitement antirétroviral }\end{array}$ \\
\end{tabular} & $\begin{array}{l}\text { Détection de l'infection } \\
\text { génitale au VHS-2 } \\
\text { Charge virale de l'infection } \\
\text { génitale au VHS-2 }\end{array}$ \\
\hline $\begin{array}{l}\text { Nagot et al. } \\
\text { (20) } 2007\end{array}$ & 140 femmes & Burkina Faso & \begin{tabular}{|l} 
Valacyclovir (500 mg bidd) par \\
rapport au placebo
\end{tabular} & 3 & \begin{tabular}{|l} 
Ne recevaient pas de \\
traitement antirétroviral
\end{tabular} & $\begin{array}{l}\text { Détection de l'infection } \\
\text { génitale au VHS-2 } \\
\text { Événements indésirables }\end{array}$ \\
\hline \begin{tabular}{|l|} 
Ouedraogo et \\
al. (21) 2006
\end{tabular} & 60 femmes & Burkina Fas & $\begin{array}{l}\text { Valacyclovir (500 mg bid) par } \\
\text { rapport au placebo }\end{array}$ & 3 & \begin{tabular}{|l|} 
Toutes les patientes \\
recevaient un \\
traitement antí́troviral \\
hautement actif \\
(HAART)
\end{tabular} & $\begin{array}{l}\text { Détection de l'infection } \\
\text { génitale au VHS-2 } \\
\text { Charge virale de l'infection } \\
\text { génitale au VHS-2 }\end{array}$ \\
\hline \begin{tabular}{|l|} 
Perti et al. (22) \\
2013
\end{tabular} & $\begin{array}{l}34 \text { hommes et } \\
\text { femmes }\end{array}$ & États-Unis & \begin{tabular}{|l} 
Acyclovir \\
(400 mg bid) par rapport au \\
valacyclovir (1000 mg bid)
\end{tabular} & 3 & 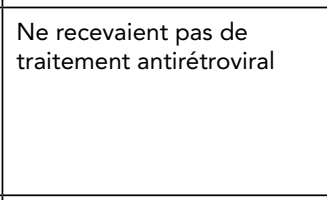 & $\begin{array}{l}\text { Détection de l'infection } \\
\text { génitale au VHS-2 } \\
\text { Charge virale de l'infection } \\
\text { génitale au VHS-2 } \\
\text { Evvénements indésirables }\end{array}$ \\
\hline $\begin{array}{l}\text { Tanton et al. } \\
\text { (23) } 2010 \text {. }\end{array}$ & 484 femmes & Tanzanie & \begin{tabular}{|l} 
Acyclovir (400 mg bid) par \\
rapport au placebo
\end{tabular} & 24 & $\begin{array}{l}\text { Non explicitement indiqué } \\
\text { (La gratuité du traitement } \\
\text { antíntroviral a éé fofferte } \\
\text { dans les hôpitaux régionaux } \\
\text { et de district dans la zone } \\
\text { d'étude durant l'essai) }\end{array}$ & $\begin{array}{l}\text { Détection de l'infection } \\
\text { génitale au VHS-2 } \\
\text { Charge virale de l'infection } \\
\text { génitale au VHS-2 } \\
\text { Evvénements indésirables }\end{array}$ \\
\hline $\begin{array}{l}\text { Tobian et al. } \\
\text { (24) } 2013 \text {. }\end{array}$ & $96^{1}$ femmes & Ouganda & $\begin{array}{l}\text { Acyclovir (400 mg bid) par } \\
\text { rapport au placebo }\end{array}$ & 24 & \begin{tabular}{|l|l|}
$\begin{array}{l}\text { Tutes les patientes } \\
\text { recevaient un traitement } \\
\text { antirítroviral }\end{array}$ \\
\end{tabular} & $\begin{array}{l}\text { Détection de l'infection } \\
\text { génitale au VHS-2 } \\
\text { Charge virale de l'infection } \\
\text { génitale au VHS-2 }\end{array}$ \\
\hline \begin{tabular}{|l|} 
Van Wagoner \\
et lal.25) 2015
\end{tabular} & $34^{2}$ femmes & États-Unis & 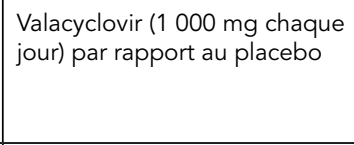 & 6 & \begin{tabular}{|l|} 
Toutes les patientes \\
recevaient un ntraitement \\
antirítroviral
\end{tabular} & $\begin{array}{l}\text { Détection de l'infection } \\
\text { génitale au VHS-2 } \\
\text { Événements indésirables }\end{array}$ \\
\hline \begin{tabular}{|l} 
Zuckerman et \\
al. (26) 2007
\end{tabular} & 20 hommes & \begin{tabular}{|l|l|l} 
Pérou \\
\end{tabular} & \begin{tabular}{|l} 
Valacyclovir $(500 \mathrm{mg}$ bidd par \\
rapport au placebo
\end{tabular} & 2 & \begin{tabular}{|l} 
Ne recevaient pas de \\
traitement antirétroviral
\end{tabular} & $\begin{array}{l}\text { Détection de l'infection } \\
\text { génitale au VHS-2 } \\
\text { Evénements indésirables }\end{array}$ \\
\hline
\end{tabular}

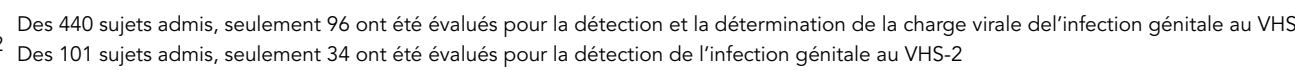


antirétroviral, la numeration de lymphocytes CD4, le nombre de cas de transmission du VHS (resultat primaire), la detection et charge virale du VHS (marqueurs de substitution du risque de

Comme il s'agissait d'une synthèse descriptive de la documentation, aucun graphique en entonnoir ni aucune évaluation statistique de l'héterogénéité n'ont été réalisés. ublié concernant cet

\section{Résultats}

Au total, 492 articles ont été répertoriés, dont 485 dans les bases de donnees et 7 au moyen des recherches des référ 315 étaient uniques. Une fois pévalination De ce nombre, '́n ection et d'inclusion terminé it restait 14 ćtudes de pour déterminer le risque de biais. Un article a été exclu en raison du risque élevé de biais (13), portant donc à 13 nombre d'articles inclus aux fins de l'examen systématique (Appendice 1).

\section{Caractéristiques de l'étude}

Les caractéristiques des 13 études sont résumées dans le Tableau 1. Seule une étude parmi les 13 a mesuré directemen la transmission du VHS (14). Les 12 autres articles portaient essentiellement sur les marqueurs de substitution du risque de nombre, 7 études traitaient à la fois de la détection duS. De ce nombre, 7 etudes traitaient à la fois de la détection du VHS et $d$ tandis que les 5 autres faisaient état uniquement de la détection $\mathrm{du} \operatorname{VHS}(16,18,20,25,26)$. Toutes les études ont eu recours à des tests de polymerase en chaine pour la detection du VHS et la détermination de la charge virale du VHS.

Les 13 essais cliniques incluaient 2367 participants co-infectés par le VIH et le VHS. La plupart des essais ont été menés auprès de populations de pays africains $(n=7)$, alors que deux essais ont été réalisés au Pérou, deux autres, aux Etats-Unis et un dernier, en Thailande. Un essai a ete mene aupres d'u échantillon de patients provenant de trois continents. La plupart des etudes ne comprenaient que des femmes $(n=9)$ masculins et que les trois autres études comprenaient des participants des deux sexes. Les périodes de suivi variaient

Tableau 2 : Sommaire des résultats comparant le traitement suppressif par l'acyclovir au placebo

\begin{tabular}{|c|c|c|c|c|c|c|c|c|}
\hline \multirow[b]{2}{*}{ Étude } & \multirow{2}{*}{$\begin{array}{l}\text { Dose } \\
\text { d'acyclovir }\end{array}$} & \multirow{2}{*}{$\begin{array}{l}\text { Traitement } \\
\text { pour le VIH }\end{array}$} & \multicolumn{3}{|c|}{$\begin{array}{l}\text { Détection de l'infection génitale au virus } \\
\text { herpès simplex (VHS) }\end{array}$} & \multicolumn{3}{|c|}{$\begin{array}{l}\text { Charge virale de l'infection génitale } \\
\text { au VHS }\end{array}$} \\
\hline & & & \begin{tabular}{|l|} 
Proportion \\
groupe de \\
traitement
\end{tabular} & \begin{tabular}{|l|} 
Proportion \\
groupe \\
placebo
\end{tabular} & $\begin{array}{l}\text { Estimation de } \\
\text { l'effet }\end{array}$ & $\begin{array}{l}\text { Groupe de } \\
\text { traitement }\end{array}$ & $\begin{array}{l}\text { Groupe } \\
\text { placebo }\end{array}$ & Valeur $p$ \\
\hline $\begin{array}{l}\text { Cowan et al., } \\
2008(16)\end{array}$ & $400 \mathrm{mg}$ bid & \begin{tabular}{|l|} 
Non \\
explicitement \\
indiqué
\end{tabular} & \begin{tabular}{|l}
$10 \%$ des \\
visites
\end{tabular} & $\begin{array}{l}23 \% \text { des } \\
\text { visites }\end{array}$ & $\begin{array}{l}R C=0,24(I C \\
\text { à } 95 \%: 0,12 \text { à } \\
0,48)\end{array}$ & & & \\
\hline $\begin{array}{l}\text { Delany et al., } \\
2009 \text { (17) }\end{array}$ & $400 \mathrm{mg}$ bid & $\begin{array}{l}\text { Ne recevaient } \\
\text { pas de } \\
\text { traitement } \\
\text { antirétroviral }\end{array}$ & $\begin{array}{l}33 \% \text { des } \\
\text { patientes }\end{array}$ & $\begin{array}{l}54 \% \text { des } \\
\text { patientes }\end{array}$ & \begin{tabular}{|l|}
$R R=0,61(I C$ \\
à $95 \%: 0,46$ à \\
$0,80)$
\end{tabular} & $\begin{array}{l}\text { Moyenne = } \\
3,38\end{array}$ & $\begin{array}{l}\text { Moyenne }= \\
3,81\end{array}$ & $p=0,13$ \\
\hline $\begin{array}{l}\text { Kim et al., } \\
2010(19)\end{array}$ & $400 \mathrm{mg}$ bid & $\begin{array}{l}\text { Ne recevaient } \\
\text { pas de } \\
\text { traitement } \\
\text { antirétroviral }\end{array}$ & \begin{tabular}{|l}
$19,4 \%$ des \\
patients
\end{tabular} & $\begin{array}{l}22,5 \% \text { des } \\
\text { patients }\end{array}$ & $\begin{array}{l}\text { Non indiqué } \\
\text { (mais } p=0,07)\end{array}$ & $\begin{array}{l}\text { Médiane = } \\
6,50\end{array}$ & $\begin{array}{l}\text { Médiane }= \\
6,90\end{array}$ & $p=0,91$ \\
\hline $\begin{array}{l}\text { Tanton et al., } \\
2010 \text { (23) }\end{array}$ & $400 \mathrm{mg}$ bid & $\begin{array}{l}\begin{array}{l}\text { Non } \\
\text { explicitement } \\
\text { indiqué }\end{array} \\
\end{array}$ & $\begin{array}{l}10,9 \% \text { des } \\
\text { visites }\end{array}$ & \begin{tabular}{|l|}
$11,8 \%$ des \\
visites
\end{tabular} & $\begin{array}{l}\mathrm{RC}=0,90(\mathrm{IC} \\
\mathrm{a} 95 \% \text { : } 0,60 \text { à } \\
1,36)\end{array}$ & $\begin{array}{l}\text { Moyenne = } \\
4,16\end{array}$ & $\begin{array}{l}\text { Moyenne }= \\
4,07\end{array}$ & $p=0,73$ \\
\hline $\begin{array}{l}\text { Tobian et al., } \\
2013(24)\end{array}$ & $400 \mathrm{mg}$ bid & \begin{tabular}{|l|} 
Toutes les \\
patientes \\
recevaient un \\
traitement \\
antirétroviral
\end{tabular} & $1,4 \%$ des visites & $\begin{array}{l}10,2 \% \text { des } \\
\text { visites }\end{array}$ & $\begin{array}{l}\mathrm{RC}=0,13 \text { (IC } \\
\text { à } 95 \%: 0,04 \text { à } \\
0,41)\end{array}$ & $\begin{array}{l}\text { Médiane }= \\
3,52\end{array}$ & $\begin{array}{l}\text { Médiane = } \\
3,57\end{array}$ & $p=0,82$ \\
\hline $\begin{array}{l}\text { Dunne et al., } \\
2008(18)\end{array}$ & $800 \mathrm{mg}$ bid & $\begin{array}{l}\text { Ne recevaient } \\
\text { pas de } \\
\text { traitement } \\
\text { antirétroviral }\end{array}$ & $\begin{array}{l}1,6 \% \text { des } \\
\text { patientes }\end{array}$ & $\begin{array}{l}42,4 \% \text { des } \\
\text { patientes }\end{array}$ & $\begin{array}{l}R R=0,00 \text { (IC à } \\
95 \%: 0,006 \text { à } \\
0,33)\end{array}$ & & & \\
\hline
\end{tabular}

Abréviations : IC, intervalle de confiance; $R C$, rapport de cotes; $R R$, risque relatifi

1 Log10 copies/mL chez les sujets ayant des taux détectables d'ADN du VHS-2 de 1 à 24 mois. Huit études comprenaient uniquement des participants qui n'avaient jamais reçu un traitement antirétroviral trois études ne comprenaient que des sujets recevant un études, il n'était pas précisé de façon explicite si les sujets recevaient ou avaient reçu un traitement antirétroviral. La plupart des essais ont comparé le traitement suppressif par l'acyclovir $(400 \mathrm{mg}$ bid ou $800 \mathrm{mg}$ bid) à un placebo $(n=7)$ ou le traitem suppressif par le valacyclovir (500 $\mathrm{mg}$ bid ou $1000 \mathrm{mg}$ chaque jour) a un placebo ( $=5)$. Une etude a comparé le traitement par le valacyclovir à doses élevées (1000 mg bid). Aucune étudi n'a utilisé le famciclovir. Tous les essais cliniques ont été évalués pour s'assurer qu'ils présentaient un faible risque de biais.

\section{Transmission du VHS-2}

Une seule étude a mesuré directement la transmission du VHS-2. Il s' agissit d' une étude bien concue dans laquelle 911 couples hétérosexuels sérodifférents ont été suivis pendant 24 mois (14). Les partenaires infectés étaient séropositifs pour le $\mathrm{VH}$ et le VHS (ne recevaient pas de traitement antirétroviral) et

(acyclovir à $400 \mathrm{mg}$ bid) ou un placebo. Les partenaires réceptifs

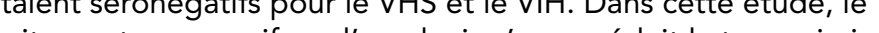
du VHS comperesiri par lacyclowir h'a pas réduin la transmission chez $9 \%(40 / 458)$ des sujets du groupe de traitement et chez $6 \%(28 / 453)$ des sujets du groupe placebo (taux de risque :

1,35 , IC à $95 \%$ : 0,83 à 2,20). Le taux de transmission du VHS des hommes aux femmes etait deux fois plus élevé que celui des

\section{Détection du VHS-2}

Douze etudes ont fait état de la détection de l'infection génitale au VHS. Ces etudes ont eu recours au test de polymérase en chaine et ont indiqué le pourcentage de participants, de visites ou de cultures/échantillons positifs pour le VHS.

\section{Acyclovir par rapport au placebo}

Six essais cliniques ayant fait état de la détection du VHS ont compare le traitement suppressif par l'acyclovir à un placebo (Tableau 2). Cinq de ces essais ont porte sur une dose normal essai a porté sur une dose plus élevée (800 mg bid) (18).

Tbleau 3 : Sommaire des résultats comparant le traitement suppressif par le valacyclovir au placebo

\begin{tabular}{|c|c|c|c|c|c|c|c|c|}
\hline \multirow[t]{2}{*}{ Étude } & \multirow[t]{2}{*}{$\begin{array}{l}\text { Dose de } \\
\text { Valacyclovir }\end{array}$} & \multirow[t]{2}{*}{$\begin{array}{l}\text { Traitement } \\
\text { pour le VIH }\end{array}$} & \multicolumn{3}{|c|}{$\begin{array}{c}\text { Détection de l'infection génitale au virus } \\
\text { herpès simplex (VHS) }\end{array}$} & \multicolumn{3}{|c|}{$\begin{array}{c}\text { Charge virale de l'infection génitale } \\
\text { au VHS }\end{array}$} \\
\hline & & & $\begin{array}{l}\text { Proportion } \\
\text { groupe de } \\
\text { traitement }\end{array}$ & $\begin{array}{c}\text { Proportion } \\
\text { groupe } \\
\text { placebo }\end{array}$ & $\begin{array}{l}\text { Estimation de } \\
\text { l'effet }\end{array}$ & $\begin{array}{l}\text { Groupe de } \\
\text { traitement }\end{array}$ & $\begin{array}{l}\text { Groupe } \\
\text { placebo }\end{array}$ & Valeur $p$ \\
\hline $\begin{array}{l}\text { Baeten et } \\
\text { al., } 2008 \\
(15)\end{array}$ & $500 \mathrm{mg}$ bid & $\begin{array}{l}\text { Ne recevaient } \\
\text { pas de } \\
\text { traitement } \\
\text { antirétroviral }\end{array}$ & $\begin{array}{l}3,7 \% \text { des } \\
\text { échantillons }\end{array}$ & $\begin{array}{l}22,1 \% \text { des } \\
\text { échantillons }\end{array}$ & $\begin{array}{l}R C=0,13(\text { IC } \\
\text { à } 95 \%: 0,07 \grave{a ̀ ~} \\
0,24)\end{array}$ & $\begin{array}{l}\text { Moyenne = } \\
3,94\end{array}$ & $\begin{array}{l}\text { Moyenne }= \\
4,80\end{array}$ & $\begin{array}{l}p= \\
0,002\end{array}$ \\
\hline $\begin{array}{l}\text { Nagot et al., } \\
2007(20)\end{array}$ & $500 \mathrm{mg}$ bid & $\begin{array}{l}\text { Ne recevaient } \\
\text { pas de } \\
\text { traitement } \\
\text { antirétroviral }\end{array}$ & $\begin{array}{l}4,1 \% \text { des } \\
\text { visites }\end{array}$ & 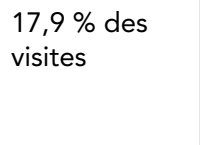 & $\begin{array}{l}R R=0,29 \\
(I C \text { a g } \\
0,14 \text { à } 0,58)\end{array}$ & & & \\
\hline $\begin{array}{l}\text { Ouedraogo } \\
\text { et al., } 2006 \\
\text { (21) }\end{array}$ & $500 \mathrm{mg}$ bid & \begin{tabular}{|l|} 
Toutes les \\
patientes \\
recevaient un \\
traitement \\
antiértroviral \\
hautement actif \\
(HAART)
\end{tabular} & $\begin{array}{l}6,6 \% \text { des } \\
\text { visites }\end{array}$ & $\begin{array}{l}9,8 \% \text { des } \\
\text { visites }\end{array}$ & $\begin{array}{l}\text { RC }=0,37(I C \\
\text { à } 95 \%: 0,13 \text { à } \\
1,05)\end{array}$ & $\begin{array}{l}\text { Moyenne }= \\
3,95\end{array}$ & $\begin{array}{l}\text { Moyenne }= \\
4,98\end{array}$ & $p=0,12$ \\
\hline $\begin{array}{l}\text { Van } \\
\text { Wagoner } \\
\text { et al., } 2015 \\
\text { (25) }\end{array}$ & $\begin{array}{l}1000 \mathrm{mg} \text { chaque } \\
\text { jour }\end{array}$ & \begin{tabular}{|l} 
Toutes les \\
patientes \\
recevaient un \\
traitement \\
antirétroviral
\end{tabular} & $\begin{array}{l}3,8 \% \text { des } \\
\text { patientes }\end{array}$ & $\begin{array}{l}12,5 \% \text { des } \\
\text { patientes }\end{array}$ & Non indiqué & & & \\
\hline $\begin{array}{l}\text { Zuckerman } \\
\text { et al., } 2007 \\
\text { (26) }\end{array}$ & $500 \mathrm{mg}$ bid & $\begin{array}{l}\text { Ne recevaient } \\
\text { pas de } \\
\text { traitement } \\
\text { antirétroviral }\end{array}$ & $\begin{array}{l}4 \% \text { des } \\
\text { échantillons }\end{array}$ & $\begin{array}{l}29 \% \text { des } \\
\text { échantillons }\end{array}$ & $\begin{array}{l}\text { Non indiqué } \\
\text { (mais } \\
p<0,001 \text { ) }\end{array}$ & & & \\
\hline
\end{tabular}

Abréviations : IC, intervalle de confiance; $R C$, rapport de cotes; $R$, risque relati

'Log10 copies/mL chez les sujets ayant des taux détectrables d'ADN du VHS-2 
Tableau 4 : Sommaire des résultats comparant le traitement suppressif par l'acyclovir au traitement suppressif par le valacyclovir

\begin{tabular}{|c|c|c|c|c|c|c|c|c|}
\hline \multirow[t]{2}{*}{ Étude } & \multirow[t]{2}{*}{ Dose } & \multirow[t]{2}{*}{$\begin{array}{l}\text { Traitement } \\
\text { pour le VIH }\end{array}$} & \multicolumn{3}{|c|}{$\begin{array}{l}\text { Détection de l'infection génitale au virus } \\
\text { herpès simplex (VHS) }\end{array}$} & \multicolumn{3}{|c|}{$\begin{array}{l}\text { Charge virale de l'infection génitale au } \\
\text { VHS }\end{array}$} \\
\hline & & & $\begin{array}{l}\text { Proportion } \\
\text { groupe } \\
\text { acyclovir }\end{array}$ & $\begin{array}{l}\text { Proportion } \\
\text { groupe } \\
\text { valacyclovir }\end{array}$ & $\begin{array}{c}\text { Estimation } \\
\text { de l'effet }\end{array}$ & $\begin{array}{c}\text { Groupe de } \\
\text { traitement } \\
\text { par l'acyclovir }\end{array}$ & $\begin{array}{l}\text { Groupe de } \\
\text { traitement par } \\
\text { le valacyclovir }\end{array}$ & Valeur $p$ \\
\hline $\begin{array}{l}\text { Perti et al., } \\
2013 \text { (22) }\end{array}$ & $\begin{array}{l}\text { Groupe } \\
\text { valacyclovir : } \\
1000 \mathrm{mg} \text { bid } \\
\text { Groupe } \\
\text { acyclovir : } \\
400 \mathrm{mg} \text { bid }\end{array}$ & $\begin{array}{l}\text { Ne recevaient } \\
\text { pas de } \\
\text { traitement } \\
\text { antirétroviral }\end{array}$ & $\begin{array}{l}8,2 \% \text { des } \\
\text { jours }\end{array}$ & $7,8 \%$ des jours & $\begin{array}{l}R R=0,95(\mathrm{IC} \\
\text { à } 95 \%: 0,66 \\
\text { à } 1,37)\end{array}$ & Médiane $=3,0$ & Médiane $=3,0$ & $p=0,67$ \\
\hline
\end{tabular}

Log10 copies/mL chez les suiet ayant des taux détectables d'ADN

Dans l'ensemble, quatre des six études ont révélé un effet statistiquement significatif du traitement suppressif par l'acyct
sur la détection du VHS, soit deux études sur des patients n'ayant jamais reçu de traitement antirétroviral $(17,18)$, une étude sur des patients ayant commencé un traitement antirétroviral au début de l'étude (24) et une étude sur des patients pour lesquels 'administration d'un traitement antirétroviral n'était pas précisée (16). La dose de $800 \mathrm{mg}$ bid semblait plus efficace que les (16). mené auprès de participants n'ayant jamais reçu de traitement antirétroviral (19) et l'autre réalisé auprès de sujets pour lesquels l'administration d'un traitement antirétroviral n'était pas précisée (23). Une seule étude parmi celles ayant évalué le traitement par mais ses résultats n'étaient pas présentés par sexe.

\section{Valacyclovir par rapport au placebo}

Cinq essais cliniques ayant fait état de la détection du VHS placemparé le traitement suppressif par le valacyclovir a un $500 \mathrm{mg}$ bid $(15,20,21,26)$, alors que l'autre étude a porté sur une dose de $1000 \mathrm{mg}$ par jour (25). Trois études (ayant toutes porté sur une dose de $500 \mathrm{mg}$ bid) ont rapporté un effet de protection statistiquement significatif du traitement suppress du valacyclovir sur la detection du VHS $(15,20,26)$, bien que lautre étude (ayant aussi porté sur une dose de $500 \mathrm{mg}$ bid) dose de $1000 \mathrm{mg}$ chaque jour) n'a pas comparé la détection du VHS entre les groupes en raison du taux limité de détection du VHS dans les échantillons des sujets (25). Dans les trois études ayant rapporté un effet significatif, tous les patients n'avaient jamais reçu un traitement antirétroviral. Les deux etudes ayan mentionne des résultats nuls (ou qui avaient eté incapables incluaient des paricipars incluaient des participants qui recevaient en concomitance un
traitement antirétroviral. Aucune des études sur le valacyclovir n'a été réalisée auprès d'un échantillon composé d'hommes et de femmes. Par conséquent, les résultats ne font pas état de différences entre les sexes.

Acyclovir par rapport au valacyclovir

Une étude a comparé directement un groupe recevant le traitement suppressif par l'acyclovir ( $400 \mathrm{mg}$ bid) à un group élevées $(1000 \mathrm{mg}$ bid). Les sujets de ces deux groupes n'avaien jamais reçu un traitement antirétroviral (22) (Tableau 4). Aucune différence significative n'a été observée en ce qui concerne la té classifiés par sexe.

\section{Charge virale du VHS-2}

Parmi les 12 études ayant fait état de la détection de l'infection virale du VHS. Ces études ont eu recours au test de polymérase en log copies/ml, dont les valeurs plus élevées semblaient indiquer la possibilité d'un risque accru de transmission.

\section{Acyclovir par rapport au placebo}

Quatre essais cliniques ayant comparé le traitement suppressif par l'acyclovir (400 mg bid) à un placebo ont fait état de la charge virale de l'infection génitale au VHS $(17,19,23,24)$ (Tableau 2). Aucune de ces études n'a révélé de différence significative entre les groupes en ce qui concerne la charge virale. Deux études incluaient des participants $n$ ayant jamais sur des patients recevant en concomitance un traitement antirétroviral (24) et une étude a été réalisée auprès de sujets pour lesquels l'administration d'un traitement antirétroviral évalué le traitement par l'acyclovir incluait des hommes et des
femmes, mais ses résultats n'étaient pas présentés par sexe. détection de l'infection génitale au VHS entre les groupes. Bien que cette étude ait inclus des sujets des deux sexes, les résultats génitale au VHS, 7 ont aussi fourni des résultats sur la charge n'était pas précisée (23). Une seule étude parmi celles ayant

\section{Valacyclovir par rapport au placebo}

Deux études ayant comparé le traitement suppressif par le valacyclovir (500 $\mathrm{mg}$ bid) à un placebo ont fait état de la charge virale de l'infection genitale au VHS (15,21) (Tableau 3). Une étude a révélé que le valacyclovir entraînait une réduction significative de la charge virale chez les participants $n$ 'ayant indiqué aucune différence significative entre les groupes d'une population recevant en concomitance un traitement antirétrovi hautement actif (HAART) (21). Aucune des études n'a fait la distinction entre les hommes et les femmes. Par conséquent, té impossible de norer des différences entre les sexes.

\section{Acyclovir par rapport au valacyclovir}

Comparativement au traitement suppressif par le valacyclovir à doses élevées (1000 mg bid), le traitement suppressif significative quant à la charge virale che les participants jamais reçu un traitement antirétroviral (22) (Tableau 4). Bien que cette étude ait inclus des sujets des deux sexes, les résultats n'ont pas été stratifiés par sexe.

\section{Événements indésirables}

\section{Acyclovir par rapport au placebo}

Parmi les sept essais cliniques ayant comparé l'acyclovir à un placebo, seulement deux ont rapporté des evenements indesirables $(17,23)$ dont les taux étaient comparables dans indésirables comprenaient des infections bactériennes non lites au traitement, des événements liés au VIH et la malaria.

Valacyclovir par rapport au placebo

Des cinq études ayant comparé le valacyclovir au placebo, quatre ont rapporté des événements indésirables. Deux études n'ont mentionné aucun événement indésirable grave sans fournir plus de détails $(15,26)$. Les deux autres études ont fait état de taux semblables d'evenements indésirables dans le groupe valacyclovir et le groupe placebo $(20,25)$. Lune de ces études courants étant les maux de tête $29 \%$ et $40 \%$ dans le groupe valacyclovir et le groupe placebo, respectivement), les réactions d'hypersensibilité $(15 \%$ et $21 \%)$, la fatigue $(15 \%$ et $25 \%$ et les nausées $(16 \%$ et $10 \%$ ) (20)

\section{Acyclovir par rapport au valacyclovir}

L'étude ayant comparé l'acyclovir au valacyclovir à doses élevées a rapporte deux evenements indésirables liés au valacyclovir à doses elevées (22). Un participant a présenté de Vurticaire, alors que deux participa

\section{Discussion}

Notre examen systématique nous a permis de répertorier une seule étude ayant examiné directement la transmission du
VHS par une population séropositive pour le VIH. Cette étude n'a toutefois révélé aucune différence significative entre le traitement suppressif par l'acyclovir et le placebo. Même si

cette étude comprenait un grand échantillon de répondants et prévoyait une longue période de suivi, cette constatation reste à confirmer. La plupart des études incluses dans cet examen rortaient essentielement sur les marqueurs de substitution du risque de transmission du VHS, qui comprenaient la détection suppressif par l'acyclovir semble efficace pour réduire la détection du VHS chez les populations séropositives pour le $\mathrm{VIH}$, mais il ne semble pas réduire efficacement la charge virale. Compte tenu des variations de l'absorption d'acyclovir (27), cette constatation contradictoire entre la détection du VHS et du traitement suppressif par le valacyclovir suourrait être lié à l'administration d'un traitement antirétroviral. Bien que cette observation ait été fondée sur un petit échantillon d'études, il semble que le traitement antirétroviral puisse annuler l'effet par ailleurs significatif du traitement par le valacyclovir sur la détection et la charge virale du VHS. Ce résultat doit toutefois être interprete avec prudence, puisqu'il pourrait être confondu puissent être à un stade plus avancé du VIH. De plus, les études incluses dans cet examen n'étaient pas conçues pour évaluer de façon précise l'effet du traitement antirétroviral.

Cet examen systématique fournit des données à jour sur un enjeu important en santé publique. Bien que les rapports
précédents aient évalué l'effet d'un traitement antirétrovira suppressif sur la co-infection au VIH et au VHS, nous n'avons pas été en mesure de traiter de façon précise de la transmission du VHS au sein d'une population co-infectée par le VIH et le VHS. L'un des principaux points forts de notre examen est la synthese des recentes publications comportant un faible risque de biais. De plus, toutes les études ont eu recours au test de polymerase du VHS, outrepassant ainsi les limites culturelles qui peuvent varier plus largement entre les études. D'après notre évaluation des publications, lorsque les cliniciens prescrivent un traitement antiviral suppressif aux patients co-infectés par le VIH et le VHS, is devraient clairement leur expliquer que le traitement ne rédu pas forcément le risque de transmission du VHS à un partenaire

D'autres efforts doivent être déployés afin de confirmer si les doses plus eleveees d'acyclovir ont un effet plus important sur la charge virale et si le valacyclovir $n$ 'est efficace que chez les populations $n$ 'ayant jamais reçu de traitement antirétroviral. En outre, bien que la résistance al 'acyclovir et au valacyclovir soit le taux de résistance est d'environ $5 \%$ dans la population séropositive pour le VIH en Amérique du Nord (27, 28). D'autres études doivent être menées chez des patients infectés par le VIH et le VHS pour déterminer l'effet d'un niveau de résistance élevé 
Les limites de cet examen comprennent la possibilité de biais de publication et l'exclusion d'essais contrôlés non randomisés. Les essais cliniques presentaicntes controles quant à la taille des traitements antirétroviraux, les périodes de suivi et les méthodes de déclaration. Même si la plupart des études n'incluaient que supérieure à 200 cellules $/ \mu \mathrm{L}$, celles-ci présentaient des variations entre elles. Les schémas posologiques des antiviraux variaie également. De plus, comme aucune étude n'a utilisé des en matière d'observance pourraient s'avérer inexas rapportes est également important de faire remarquer que toutes les études qui se sont penchées sur la détection et la charge virale disposaient de la puissance statistique pour évaluer les résultats liés au VIH, plutôt que les résultats présentant un intérêt aux fins de cet examen. Par conséquent, il est possible que, dans certains cas, les tailles des échantillons aient eté trop petites ou la charge virale du VHS. Finalement, la plupart des études ont été menées dans des pays en développement auprès de populations hétérosexuelles. La généralisabilité de ces résultats a un contexte canadien et aux populations d'hommes ayant des relations sexuelles avec d'autres hommes peut être limitée.

\section{Conclusion}

Bien que les traitements antiviraux suppressifs puissent réduire la détection et la charge virale du VHS chez les sujets co-infectés par le VIH, leur effet sur la transmission du VHS reste à confirme. Lorsqu'ils prescrivent un traitement antiviral suppressifaux patients co-infectés par le VIH et le VHS, les cliniciens doivent transmission du VHS aux partenaires réceptifs.

\section{Remerciements}

Les auteurs aimeraient remercier Shalane Ha pour son travail en tant qu'examinateur tiers en ce qui concerne l'évaluation que la bibliothécaire de portefeuille, Ella Westhaver, pour son aide quant à la recherche systématique et de l'extraction de la documentation. Les auteurs aimeraient également remercier Margaret Gale-Rowe, Karen Timmerman, Jun Wu et

\section{Conflit d'intérêts}

II n'y a aucun conflit d'intérêts à déclarer.

\section{Financement}

Aucun financement n'a été reçu pour cette étude.
Appendice 1 : Organigramme de la sélection des essais controlés randomisés aux fins d'exame

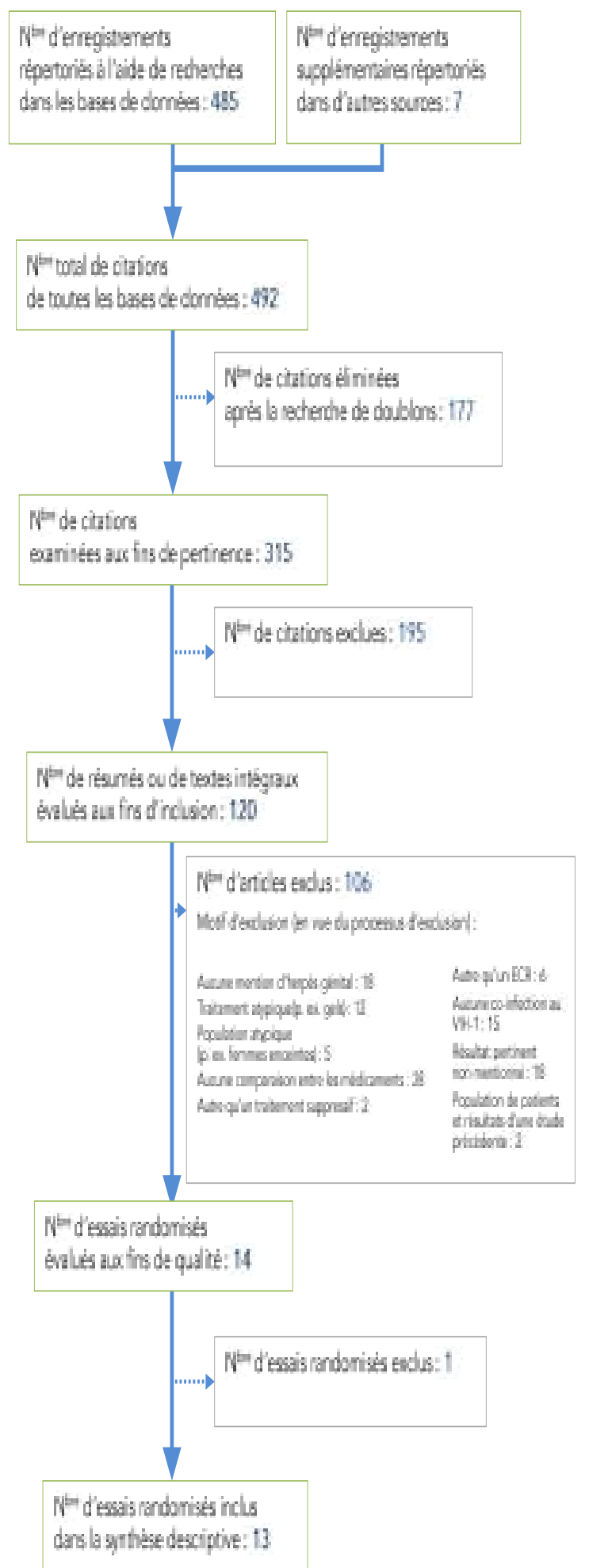

\section{Références}

1. Rotermann $M$, Langlois $K A$, Severini $A$, Totten $S$. Prevalence of Chlamydia trachomatis and herpes simplex virus type Survey. Health Rep. 2013;24(4):10-5.

2. Smith JS, Robinson NJ. Age-specific prevalence of infection with herpes simplex virus types 2 and 1: a global review. $J$ with herpes simplex virus types 2 and
Infect Dis. 2002;186 (Suppl 1):S3-28.

3. Freeman EE, Weiss HA, Glynn JR, Cross PL, Whitworth JA, Hayes RJ. Herpes simplex virus 2 infection increases HIV acquisition in men and women: systematic review and
analysis of longitudinal studies. AIDS. 2006;20:73-83.

4. Van de Perre P, Segondy M, Foulongne V, Ouedraogo A, Konate I, Huraux JM, et al. Herpes simplex virus and HIV-1: deciphering viral synergy. Lancet Infect Dis. 2008;8:490-7.

5. Schacker T, Zeh J, Hu HL, Hill J, Corey L. Frequency of symptomatic and asymptomatic HSV-2 reactivations
HIV-infected men. I Infect Dis. 1998; 178:1616-22.

6. Augenbraun M, Feldman J, Chirgwin K, Zenilman J, Clarke L, DeHovitz J, et al. Increased genital shedding of herpes simplex virus type 2 in HIV-seropositive women. Ann Intern

Freeman EE, Weiss HA, Glynn JR, Cross PL, Whitworth JA, Hayes RJ. Herpes simplex virus 2 infection increases HIV acquisition in men and women. systematic review and me

8. Mertz GJ. Asymptomatic shedding of herpes simplex virus 1 and 2: implications for pre
Dis. 2008:198(8):1098-100.

9. Corey L, Wald A, Patel R, Sacks SL, Tyring SK, Warren T, et al. Valacyclovir HSV Transmission Study Group. Once-daily herpes. N Engl J Med. 2004;350(1):11-20.

10. Ruddock $B$, Severn $M$; Health Technology Inquiry Service. Oral antivirals for the treatment and prevention of orolab and genital herpes. The Health Technology Inquiry Service.
Ottawa (ON): Canadian Agency for Drugs and Technologies in Health; 2007.

11. Jungmann EM. Genital herpes. BMJ Clin Evid. 2007;2007:1603.

12. Higgins JP, Altman DG, Gøtzsche $P C$, Jüni $P$, Moher $D$, Oxman AD, et al. The Cochrane Collaboration's tool for assessing risk
2011;343:d5928.

13. Nijhawan AE, DeLong AK, Chapman S, Rana A, Kurpewski $J$, Ingersoll J, et al. Effect of HSV-2 suppressive therapy on genital tract HIV-1 RNA shedding among women on HAART: a pilot randomized controlled trial. Infect Dis Obstet

14. Mujugira $A$, Magaret AS, Celum $C$, Baeten JM, Lingappa $J R$, Morrow RA, et al. Daily acyclovir to decrease herpes coinfected persons: a randomized controlled trial. J Infect

15. Baeten JM, Strick LB, Lucchetti A, Whittington WL, Sanchez , Coombs RW, et al. Herpes simplex virus (HSV)-suppressive therapy decreases plasma and genital HV-1 levels in HSV-2/ cross-over trial. J Infect Dis. 2008;198(12):1804-8.

16. Cowan $F M$, Pascoe $S J$, Barlow $K L$, Langhaug $L F$, Jaffar $S$, Hargrove JW, et al. A randomised placebo-controlled trial
to explore the effect of suppressive therapy with acyclovir on genital shedding of HIV-1 and herpes simplex virus type 2 among Zimbabwean sex workers. Sex Transm Infect. 2008;84(7):548-53.

17. Delany S, Mlaba N, Clayton T, Akpomiemie G, Capovilla A, Legoff J, et al. Impact of aciclovir on genital and plasma HIV-1 RNA in HSV-2/HIV-1 co-infected women: a randomised placebo-controlled trial in South Africa. AIDS.

18. Dunne EF, Whitehead $S$, Sternberg M, Thepamnuay $S$, Leelawiwat W, McNicholl JM, et al. Suppressive acyclovir therapy reduces HV cervicovaginal shedding in HIV- and
HSV-2-infected women, Chiang Rai, Thailand. J Aca Immun Defic Syndr. 2008;49(1):77-83.

19. Kim HN Wang J Hughes J, Coombs R, Sanchez J, Reid S, et al. Effect of acyclovir on HIV-1 set point among herpes simplex virus type 2-seropositive persons during early HIV-1 infection. J Infect Dis. 2010;202(5):734-8.

20. Nagot $N$, Ouédraogo $A$, Foulongne $V$, Konaté I, Weiss $H A$, Vergne $L$, et al. Reduction of HN- RNA levels with therapy to suppress
2007;356(8):790-9.

21. Ouedraogo A, Nagot N, Vergne L, Konate I, Weiss HA, Defer $M C$, et al. Impact of suppressive herpes therapy on genital HIV-1 RNA among women taking antiretroviral therapy: a

22. Perti T, Saracino M, Baeten JM, Johnston C, Diem K, Ocbamichael N, et al. High-dose valacyclovir decreases plasma HIV-1 RNA more than standard-dose acyclovir in crossover trial. J Acq Immun Def Synd. 2013:63(2):201-8.

23. Tanton C, Weiss HA, Rusizoka M, LeGoff J, Changalucha J, Baisley $K$, et al. Long-term impact of acyclovir suppressive women: a randomized controlled trial. J Infect Dis. 2010;201(9):1285-97.

24. Tobian AA, Grabowski MK, Serwadda D, Newell K, Ssebbowa P, Franco $V$, et al. Reactivation of herpes simplex virus type 2 after initiation
Dis. 2013;208(5):839-46.

25. Van Wagoner N, Geisler WM, Bachmann LH, Hook EW. The effect of valacyclovir on HIV and HSV-2 in HIVinfected persons on antiretroviral therapy with previously
unrecognised HSV-2. Int J STD AIDS. 2015;26(8):574-81.

26. Zuckerman RA, Lucchetti A, Whittington WL, Sanchez J,

Coombs RW, Zuñiga R, et al. Herpes simplex virus (HSV) 
suppression with valacyclovir reduces rectal and blood plasma HIV-1 levels in HIV-1/HSV-2-seropositive men: a randomized, double-blind, placebo-controlled crossover trial. J Infect Dis. 2007;196(10):1500-8.

27. Reyes M, Shaik NS, Graber JM, Nisenbaum R, Wetherall NT, Fukuda K, et al. Acyclovir-resistant genital herpes among persons attending sexually transmitted disease and human immunodeficiency virus clinics. Arch Intern Med. 2003;163(1):76-80.

28. DeJesus E, Wald A, Warren T, Schacker TW, Trottier S, Shahmanesh $\mathrm{M}$, et al. Valacyclovir for the suppression of recurrent genital herpes in human immunodeficiency virusinfected subjects. J Infect Dis. 2003;188(7):1009-16. 